\title{
Narrative games in ergodic media
}

\author{
Miguel Carvalhais \& Pedro Cardoso \\ INESCTEC \& Faculty of Fine Arts, University of Porto \\ E-mail: mcarvalhaisefba.up.pt / pcardosoefba.up.pt
}

\begin{abstract}
Computational media allow the development of very particular relationships with readers. Their nature allows them to register static information but also complex and contingent behaviours that they are capable to operationalise, thus becoming interactive and immersive. These media exist in a dual state between a surface layer and a subface layer. These two are inextricably connected, with the subface often becoming a black box that can only be peered at through surface effusions that both mediate and iso-

discovered through a process of virtuosic interpretation that allows readers to form a theory of system, breeding empathy with it, and ultimately, transferring some of its processes to their minds. This paper focuses on how virtuosic interpretation is developed, and how from it stems the development of a unique kind of aesthetic experience. It explores how computational media, through anamorphosis and a dialectics of aporia and epiphany, become narrative games.
\end{abstract} late it. The procedural layer of the subface can be

\section{On Ergodic Media}

COMPUtATIOnAL media are often defined vaguely. Any media form that is processor- or process-based may fit this description, but they are often also described using a range of falsely equivalent terms such as digital media, software-based media, computer media, algorithmic media, new media, etc.. We prefer to define these forms as computational media, and the artworks produced with them as computational artworks, as systems in which computation is fundamental in their creation or development. Computation, itself an often loosely defined term, is any "process that obeys finitely describable rules." (Rucker, 2005, p. 11) This may of course mean that digital computers are used in a work, but the term does not exclude works that do not resort to program code, algorithms of effective procedures (Weizenbaum, 1976, p. 46), but that rely instead on processes and computation as a "representational medium for describing structure and process." (Mateas, 2005)

Data de submissão: 2017-12-17. Data de aprovação: 2018-07-19.

A Revista Estudos em Comunicação é financiada por Fundos FEDER através do Programa Operacional Factores de Competitividade - COMPETE e por Fundos Nacionais através da FCT - Fundação para a Ciência e a Tecnologia no âmbito do projeto Comunicação, Filosofia e Humanidades (LabCom.IFP) UID/CCI/00661/2013.
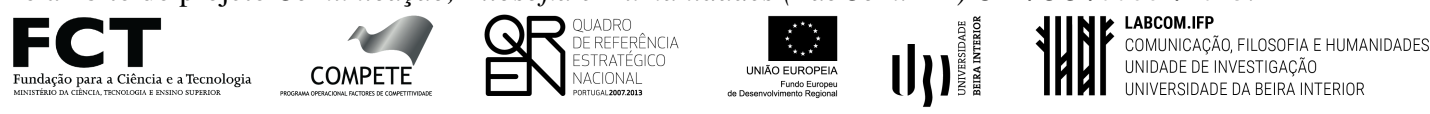
In these media forms, computation is not only used because of its immediate qualities, but rather because it is understood to be meaningful, becoming one of the central aesthetic outputs of an artwork.

Computational is therefore not strictly a technological description, as the resources used in a given work can be classical, or non-computational in themselves, as we can find in e.g. Raymond Queneau's Cent Mille Milliards De Poèmes (1961), Marc Saporta's Composition $N^{o} 1$ (1962), or in many of Zimoun's installations. ${ }^{1}$ Computational here describes the processing of information, but the machine developing it does not need to be physically instantiated in the artwork itself, as it can be instantiated in the human reader.

What seems to be fundamental is how artworks are defined by computation and how this affects their nature and that of the relations humans ${ }^{2}$ establish with them. If we prefer to describe these media forms in a way that is more focused on these relations than on their technological substrate, we may also a term proposed by Espen Aarseth (1997) and call them ergodic. ${ }^{3}$

\section{Black Boxes}

Computational technologies are ubiquitous and pervasive. As a universal solvent for media (Hayles, 2005) they have been replacing many technologies in our lives and increasing the frequency of our contacts with ergodic media forms. As a growing number of media forms are created, distributed and experienced in computational contexts, both classical forms - as novels, films, etc. - and forms that are native to these media - as videogames, interactive fictions, etc. - start sharing several traits. Although some links with historical models or patterns tend to be preserved in this process - a phenomenon that breeds several cases of remediation or skeuomorphism ${ }^{4}$ along the way - the effects of the "procedural attractor" ${ }^{5}$ in the digital medium ${ }^{6}$ are very strong and as such.

1. http://zimoun.net/

2. Regardless of each individual human's stance towards the work, e.g. as author, or reader. We could describe the human counterparts in these processes using terms as users, interactors, or players but, as none of these terms adequately describes them in all contexts, we have preferred to use the more generic term reader.

3. Although this term also exists in physics and mathematics, Aarseth defines it based on its etymology, from "the Greek words ergon and hodos, meaning 'work' and 'path'." (1997, p. 1). He uses it to describe texts in which the reader is required to do an amount of "nontrivial effort" to traverse them, where users effectuate "a semiotic sequence, and this selective movement is a work of physical construction that the various concepts of 'reading' do not account for." (1997, p. 1) This ergodic level of a medium is developed concurrently with other levels, such as description or narration.

4. Skeuomorphism is the intentional imitation of features from other materials or artefacts in the design of an artefact. Common examples can be found in the mimicking of physical controllers in user interfaces, "old-style mechanical representations in our new digital environments" (Cooper et al., 2014).

5. A term we derive from the mathematical concept of attractor in dynamical systems, the set of states to which a system tends to evolve regardless of the wide variety of starting conditions it may have (Lorenz, 1995; DeLanda, 1997). This concept was quickly repurposed in other areas of knowledge, e.g. the study of morphogenesis both in natural as in artificial systems (Alexander, 2002; Ball, 2004). As in computational media procedurality is the strongest of affordances, even media artefacts designed to explicitly simulate characteristics of classical media as e.g. linearity, tend to become procedural and to break away from those constraints.

6. Janet Murray argues that computational technologies allow the emergence of a Digital Medium that very often seems plural to us, as it breeds so many apparently diverse forms (Murray, 2003, p. 3). 
As media forms shift to computational contexts, contingent and complex behaviours become not only possible but almost inevitable. As new modes of communication become attainable, media forms become more participatory. As they develop complex topologies - because of the scale of information but also due to the ways how this information is presented - they become more spatial and encyclopaedic (Murray, 2012).

Therefore, all communication developed with these systems is characterised by a duality between surface and subface (Nake, 2016). The surface is the analogue sensorial layer of a medium, through which signs can be communicated to humans. The subface is the immaterial, algorithmic, substrate of a medium, where computation, code, and processes are developed.

We do not usually have access to the subface. It is hidden, internal to the computer or the software system. (...) In ordinary terms, we may say that the subface is the algorithm, the description of the class, the program-and-data. In the same manner of describing the situation, the surface is the image on screen, in projection, be it still or dynamic, passive or interactive. (Nake, 2016, p. 16)

The subface is inextricably linked to a surface that mediates it, but because readers are unable to directly access it, it is also inevitably secluded. ${ }^{7}$ The subface therefore becomes a black box (Minsky, 1972, p. 32), a system that readers can only explore through its outputs, its surface effusions. But if with many black boxes one may not be interested so much in their inner workings as on their results or outputs, in computational artworks the mechanics of the black box become fundamental to understand.

\section{Aesthetics of Mechanics}

The mechanics layer of a computational system is defined by the MDA ${ }^{8}$ Framework as "the particular components of the game, at the level of data representation and algorithms." (Hunicke, LeBlanc, \& Zubek, 2004) The "run-time behavior of the mechanics acting on player inputs and each others' outputs over time" is then described as the system's dynamics, which in turn will give rise to the layer of aesthetics, that of the "emotional responses evoked" in the readers during the contact with the system.

The MDA framework was developed to study videogames, with its fundamental focus resting on the "idea that games are more like artifacts than media", i.e. that their central content is behaviour, "not the media that streams (...) towards the player." MDA therefore becomes relevant to the study of other computational and ergodic forms precisely because of how it establishes each of these layers as a "lens" or a "view" of the system, separate from one another but causally linked, both from the designer's as from the reader's points of view.

7. We could argue that even in systems that give readers direct access to representations of program code, the subface is still being mediated by a surface, in this case the representation of the subface as program code that still needs to be interpreted or compiled. The subface is therefore not the program code in itself, but rather those processes that are instantiated in the computational machine, and although these are only represented by the program code, they are not the program code.

8. MDA stands for Mechanics, Dynamics, and Aesthetics. 
From the player's perspective, aesthetics set the tone, which is born out in observable dynamics and eventually, operable mechanics. (Hunicke, LeBlanc, \& Zubek, 2004)

If dynamics are observable by reader, the layer of mechanics is usually not directly perceivable. However, in many ways it is at least as relevant to the aesthetic experience as the dynamics. We should be careful to not understand mechanics as synonymous with subface, and dynamics as synonymous with surface. We believe that what Nake describes as subface includes the layer of mechanics but also intersects that of dynamics, while the surface starts at the level of the dynamics and overlaps aesthetics but does not include the entirety of this last layer - as MDA's aesthetics also includes phenomena that are exogenous to the artwork, and internal to the human.

In open-ended systems that are able to generate seemingly endless outputs and variations, if the reader only follows the surface of the system, they may be confronted with something that may very well be infinite, or at least potentially infinite at a human scale. In these cases, regardless of how much effort and time may be put into reading a system, no human will ever be able to fully exhaust its outputs. As two examples of this we may consider the 10^14 different sonnets that Raymond Queneau's Cent Mille Milliards De Poèmes (1961) is able to generate, or the $1.8 \mathrm{x}$ $10 \wedge 308$ unique icons that John F. Simon Jr.'s Every Icon (1997) is able to produce, magnitudes that are impossible to be experienced to completeness during an entire human lifespan. Furthermore, even if every single of these outputs is new, their surprise and value to any given reader tend to decrease proportionally to the total number of outputs perceived (Boden, 2004). Therefore, a full and complete reading of all the outputs is not only impossible, as it is also not intended. What then constitutes the focus of the aesthetic experience of these artworks? And how are readers able to experience closure in their reading?

We propose that the aesthetic enjoyment of computational works is deeply linked with the understanding of their subface. Signs produced by computational systems have a dual nature, and although the relationship between subface processes and surface signs is arbitrary - i.e. not being constrained in principle by the physical materiality of any given medium or material (Aarseth, 1997, p. 40) - the surface level remains fundamental. This happens for two reasons. The first is how often it is the focus of aesthetic enjoyment, with surface signs being appreciated per se, for the forms, rhythms, narratives, etc. that are created and conveyed. The second is how surface signs are often the only gateway available for readers to understand what may be going on at the internal level of the subface. If the subface ontologically precedes the surface, setting the field for everything that may happen there and that can only be "fully understood (...) in light of the internal" level, it is also true that from the reader's point of view, whatever is at the subface level "can only be fully experienced by way of the external, expressive level" (Aarseth, 1997, p. 40) of the surface.

If we cannot exhaust surface signs, understanding the subface may allow us to anticipate an artwork's formal development, its behaviours and outputs. In interactive systems, it will also allow us to understand the "repertoire of possible steps and rhythms" and to "improvise a particular dance among the many, many possible dances the author has enabled" (Murray, 1997, p. 153). 
Once we realise that these works are not merely objects but rather machines that may evolve autonomously from the reader, ${ }^{9}$ the development of a degree of empathy with them, of a model or mental simulation of its subface, of what we may call a Theory of the System (ToS), ${ }^{10}$ may allow readers to develop a procedural understanding of the artefact, and through this, to attain an experience of closure.

It should be noted that this ToS is often not a full mental simulation of the system's mechanics. We prefer to think of it as an intuitive understanding of the subface, a collection of mental models of the system (Ramachandran, 2011), of heuristics that help to foresee behaviours (Norman, 2007; Murtaugh, 2008), and not as a formal description of its mechanics or of its actual code. Furthermore, we do not follow or model all the computational processes in an artwork. What we follow are those processes that are perceived to be relevant from causal and aesthetic points of view. Many other processes, perhaps even the majority of the processes that constitute an artwork, are perfunctory or transparent, and as such do not need to be considered.

\section{Reading Processes}

If understanding the subface is fundamental to aesthetic enjoyment, and the surface is the only entrance point we have to the subface, it follows that reading these systems becomes more than the interpretation of their surface signs but also the exercise of inferring the subface from the surface. How can this process be developed?

Some artworks can be seen as white boxes, because they present readers with code or a pseudocode description of the processes in the subface. An example of these procedural descriptions can be found in Every Icon (1997), an iterative process shown on a LCD screen and accompanied by the following text ${ }^{11}$ :

Given: An icon described by a $32 \times 32$ grid.

Allowed: Any element of the grid to be colored black or white.

Shown: Every icon.

Some works show actual code, as Pall Thayer's Microcodes series, from which Sleep (2009) presents itself as two lines of Perl:

\#!/usr/bin/perl

sleep $((8 * 60) * 60)$;

9. Regardless of whether interaction is part of the process or not.

10. A concept we derive from the Theory of Mind (ToM), the capacity to build mental models of other persons' minds (Metzinger, 2009; Ramachandran, 2011), to "imagine the mind of others" (Dehaene, 2009). It is ToM that allows us to "be aware of such mental states as desires and intentions in both ourselves and others" (Shermer, 2011, p. 87 ) and to become able to attribute agency to others (both humans, as animals and other objects, artefacts, or systems). ToM is engaged by many narrative forms, such as the theatre of novels, where we "construct [...] mental states from the observable actions and from what the protagonists choose to report to us." (Zunshine, 2006)

11. Simon does not present the actual code but summarises it in three phrases. "If you can think like a programmer, you can reconstruct the binary formula behind his marching pixels, but Simon's paraphrase is so guileless that lay viewers can get a feeling for the dynamic as well.” (Blais \& Ippolito, 2006, p. 25) 
Casey Reas's Process series are presented as artefacts - sometimes dynamic, other times static - that are accompanied by pseudo-code descriptions of elements such as:

Element 1

Form 1: Circle

Behavior 1: Move in a straight line

Behavior 2: Constrain to surface

Behavior 3: Change direction while touching another element

Behavior 4: Move away from an overlapping element

And processes, such as:

Process 4

A rectangular surface filled with varying sizes of Element 1. Draw a line from the centers of Elements that are touching. Set the value of the shortest possible line to black and the longest to white, with varying grass representing values in between.

But white box systems are rare. Not only they demand users to be able to understand the code or its descriptions, as they may quickly turn into black box systems due to the complexity of the processes that are presented. Even if a reader is able to grasp the entirety of the procedural descriptions and is able to imagine their deployment, beyond what seems to be a low threshold of complexity, its development may very well be impossible to follow. If the processes of Every Icon or Sleep are easy to work out to their ultimate consequences, allowing readers to either compute them step by step or quickly imagine all of their possible formal outputs, ${ }^{12}$ those of Process 4 (2005) are far more complex, and even if a reader understands the descriptions, they will almost inevitably find it difficult to predict their development. Given some of the clues in the pseudocode, regarding e.g. colours or shapes, some understanding of the field of possibilities will be attained, but this is probably not enough to anticipate the actual formal and behavioural outputs of the system.

If a reader is faced with an interactive black box system, they may be able to directly peruse its surface and through interaction try to probe the subface, trying to understand its mechanics through direct testing (Lopes, 2010; Kwastek, 2013). But even direct interaction may not suffice to grasp the subface, and furthermore, not all systems are interactive. Therefore, how is the surface read in search of the subface?

The process we describe as virtuosic interpretation (Carvalhais \& Cardoso, 2015b, 2017) starts, like every reading act, with the interpretative user function (Aarseth, 1997, p. 62), through which information starts flowing from the surface to the reader. This interpretative function is omnipresent, and is found not only in ergodic forms as well as in all other media forms, where it is the only function accessible to readers and as such very often dominant (Bogost, 2006, p. 108). Ergodic forms are characterised precisely for allowing the development of further user functions,

12. Or, to be more precise, to understand the complete field of possibilities within which the system's behaviours will happen and the outputs be generated (Upton, 2015), and to imagine possible articulations to be developed inside this field, which is to say, consequently, to also imagine all the behaviours and outputs that the system will not be likely or able to produce. 
such as the explorative and the configurative functions (Aarseth, 1997, p. 62; Carvalhais, 2016, p. 244). Through the first of these a reader will be able to decide which paths to follow along the experience of a system, while through the second they will manage to select or create new surface units within the system.

The balance between functions may vary depending on the dynamics of a system, and whether it allows for direct interaction. In non-interactive systems, or in vicarious interaction (Kwastek, 2013, p. 94; Carvalhais \& Cardoso, 2015a), the interpretative function will take the lead role, but whenever interaction is possible, any of the other functions may lead. ${ }^{13}$ The procedural modality (Strickland, 2007; Carvalhais, 2016), the human penchant for pattern detection (Eagleman, 2011; Shermer, 2011, p. 5) and for identifying intentional stances and causal relations and affinities (Pinker, 1999), will nevertheless still allow us to develop the explorative and configurative functions even in non-interactive systems. However, these are not directly developed in or with the system.

As surface signs are read and causal relations deduced, the reader will amass information. Mechanics previously experienced in other systems may also be adapted to the current context. ${ }^{14}$ From all these, the reader will be able to develop a tentative mental model of the system, a conjectural ToS. This ToS can then be iteratively refined through confrontation with the actual system, which will allow the confirmation, fine-tuning, or falsification of the hypotheses therein encapsulated.

This effort is dependent on the reader's capability to deduce, remember, and repurpose processes, but also on the possibility to establish multiple contacts with the system, so that the ToS may be evaluated. ${ }^{15}$

This is a gradual process of creation of meaning. The interpretation of signs, behaviours, and processes is by and large a subconscious process of learning, of building of knowledge about the system and of progressively reducing the uncertainty about its behaviour. It is a process of gradually getting to understand the machine through direct contact with its surface effusions or indirect contact with its simulations in the ToS.

\section{Towards Empathy}

As a consequence of virtuosic interpretation, an artwork becomes more than its layers of mechanics, dynamics, and aesthetics, being expanded by two further layers that are developed mentally by the reader and are continuously confronted with it: a layer of simulated dynamics, and one of simulated mechanics.

The system thus becomes more than just its surface representations and the particular computational instantiation one is confronted with. As a reader develops empathy with the system, some of its mechanics are effectively transferred to the reader's mind, where processes may continue to

13. This is often the case with games or with game-like experiences.

14. These may include mechanics from physics, and other real-life examples that may be analogue in any way to the system being read.

15. The capability to establish multiple contacts can greatly vary, ranging from the trivial, with dynamic generative systems, to the very difficult, with analogue outputs from computational systems as e.g. prints, silkscreens, plots, 3D prints or other media. In cases such as these, virtuosic interpretation may nevertheless be possible to develop, although at a level far more removed from the actual computational artefact. 
be developed far beyond the duration of the actual contact. We can then regard the dissemination or replication of a work's procedural foci to human minds as one of the goals of computational artworks, perhaps their quintessential goal. ${ }^{16}$

Furthermore, during the process of virtuosic interpretation, this trial-and-error stage of developing a ToS, the reader is as likely to find models capable of producing good previsions as of running into models that do not and that are quickly falsified when confronted with the artwork. Finding that a model is false or incorrect will allow for its revision and correction but will inevitably also lead to an experience of aporia (Aarseth, 1997, p. 91). Conversely, the confirmation of a model through the verification of its predictions will lead the reader to experiences of epiphany.

This aporia-epiphany dynamic is conducive to the development of a unique kind of aesthetic experience that resorts to cognitive processes not often explored in other media. Not being in itself a narrative structure, this dynamic "constitutes a more fundamental layer of human experience, from which narratives are spun." (Aarseth, 1997, p. 92) As such, computational media become narrative games that involve the reader in processes of anamorphosis by leading them to assume unconventional stances towards the medium, and ultimately to forfeiting the original medium altogether, once that the processes are transferred through virtuosic interpretation and the development of a ToS.

\section{Acknowledgements}

FourEyes is a Research Line within project "TEC4Growth - Pervasive Intelligence, Enhancers and Proofs of Concept with Industrial Impact/NORTE-01-0145-FEDER-000020" financed by the North Portugal Regional Operational Programme (NORTE 2020), under the PORTUGAL 2020 Partnership Agreement, and through the European Regional Development Fund (ERDF).

\section{References}

Aarseth, E. J. (1997). Cybertext: Perspectives on Ergodic Literature. Baltimore, MD: The Johns Hopkins University Press.

Albert, S. (2009). Artware. In J. B. Slater \& P. v. M. Broekman (eds.), Proud to Be Flesh: A Mute Magazine Anthology of Cultural Politics After the Net (pp. 89-92). London: Mute Publishing.

Alexander, C. (2002). The Nature of Order: An Essay on the Art of Building and the Nature of the Universe. Book Two: The Process of Creating Life. Berkeley, CA: The Center for Environmental Structure.

16. We could establish a parallel with conceptual art, given that the main goal of both forms seems to be the transmission of concepts, procedures or instructions (Albert, 2009). Both in conceptual art as in computational art, very often the artist's attention "is focused on exploring systems for their own intrinsic value" (Galanter, 2003, p. 18) but in computational art, code and processes are almost never communicated directly to the reader but are rather mediated by artefacts that embody and instantiate it, that mediate it through computation. Therefore, computational art so becomes because it communicates computation through computation, expressing computation. 
Ball, P. (2004). The Self-Made Tapestry: Pattern Formation in Nature. Oxford: Oxford University Press.

Berry, D. M. (2011). The Philosophy of Software: Code and Mediation in the Digital Age. Basingstoke: Palgrave Macmillan.

Blais, J., \& Ippolito, J. (2006). At the Edge of Art. London: Thames \& Hudson.

Boden, M. A. (2004). The Creative Mind: Myths and Mechanisms. (Second ed.). London: Routledge.

Bogost, I. (2006). Unit Operations: An Approach to Videogame Criticism. Cambridge, MA: The MIT Press.

Carvalhais, M. (2016). Artificial Aesthetics: Creative Practices in Computational Art and Design. Porto: U.Porto Edições.

Carvalhais, M. \& Cardoso, P. (2015a). Beyond Vicarious Interactions: From Theory of Mind to Theories of Systems in Ergodic Artefacts. Paper presented at $x \operatorname{CoAx} 2015$, Glasgow. http://2015.xcoax.org/pdf/xcoax2015-Carvalhais.pdf.

Carvalhais, M. \& Cardoso, P. (2015b). What Then Happens When Interaction is Not Possible: The Virtuosic Interpretation of Ergodic Artefacts. Journal of Science and Technology of the Arts, 7(1): 55-62. doi: 10.7559/citarj.v7i1.144.

Carvalhais, M. \& Cardoso, P. (2017). Creation of Meaning in Processor-based Artefacts. Paper presented at ISEA 2017, Bio-Creation and Peace, Manizales.

Cooper, A.; Reimann, R.; Cronin, D. \& Noessel, C. (2014). About Face: The Essentials of Interaction Design, Fourth Edition. Indianapolis, IN: Wiley.

Dehaene, S. (2009). Reading in the Brain: The Science and Evolution of a Human Invention. New York, NY: Viking.

DeLanda, M. (1997). A Thousand Years of Nonlinear History. Brooklyn, NY: Zone Books.

Eagleman, D. M. (2011). Incognito: The Secret Lives of the Brain. New York, NY: Pantheon Books.

Galanter, P. (2003). What is Generative Art? Complexity theory as a context for art theory. Paper presented at the Generative Art, Milan. www.philipgalanter.com/downloads/ga2003_what_is _genart.pdf

Hayles, N. K. (2005). My Mother Was a Computer: Digital Subjects and Literary Texts. Chicago, IL: The University of Chicago Press.

Hunicke, R.; LeBlanc, M. \& Zubek, R. (2004). MDA: A formal approach to game design and game research. Paper presented at the Challenges in Games AI Workshop, Nineteenth National Conference of Artificial Intelligence, San Jose, CA.

Kwastek, K. (2013). Aesthetics of Interaction in Digital Art (N. Warde, Trans.). Cambridge, MA: The MIT Press.

Lopes, D. M. (2010). A Philosophy of Computer Art. Oxon: Routledge. 
Lorenz, E. N. (1995). The Essence of Chaos. Seattle, wA: University of Washington Press.

Mateas, M. (2005). Procedural Literacy: Educating the New Media Practicioner. On The Horizon. Special Issue: Future of Games, Simulations and Interactive Media in Learning Contexts, 13(1).

Metzinger, T. (2009). The Ego Tunnel: The Science of the Mind and the Myth of the Self. New York, NY: Basic Books.

Minsky, M. (1972). Computation: Finite and Infinite Machines. London: Prentice-Hall International.

Murray, J. H. (1997). Hamlet on the Holodeck: The Future of Narrative in Cyberspace. Cambridge, MA: The MIT Press.

Murray, J. H. (2003). Inventing the Medium. In N. Wardrip-Fruin \& N. Montfort (eds.), The New Media Reader (pp. 3-11). Cambridge, MA: The MIT Press.

Murray, J. H. (2012). Inventing the Medium: Principles of Interaction Design as a Cultural Practice. Cambridge, MA: The MIT Press.

Murtaugh, M. (2008). Interaction. In M. Fuller (ed.), Software Studies: A Lexicon (pp. 143-149). Cambridge, MA: The MIT Press.

Nake, F. (2016). The Disappearing Masterpiece. In M. Verdicchio, A. Clifford, A. Rangel, \& M. Carvalhais (eds.), xCoAx 2016: Proceedings of the fourth conference on Computation, Communication, Aesthetics, and X. (pp. 11-26). Bergamo.

Norman, D. A. (2007). The Design of Future Things. Philadelphia, PA: Basic Books.

Pinker, S. (1999). How the Mind Works. London: Penguin Books.

Queneau, R. (1961). Cent Mille Milliards de Poèmes. Paris: Gallimard.

Reas, C. E. B. (2005). Process 4.

Ramachandran, V. S. (2011). The Tell-Tale Brain: A Neuroscientist's Quest for What Makes Us Human. New York, NY: W. W. Norton \& Company.

Rucker, R. (2005). The Lifebox, the Seashell, and the Soul: What Gnarly Computation Taught Me About Ultimate Reality, the Meaning of Life, and How to Be Happy. New York, NY: Thunder's Mouth Press.

Shermer, M. (2011). The Believing Brain: From Ghosts and Gods to Politics and Conspiracies How We Construct Beliefs and Reinforce Them as Truths. New York, NY: Times Books.

Simon, J. F., Jr. (1997). Every Icon.

Strickland, S. (2007). Quantum Poetics: Six Thoughts. In E. Kac (ed.), Media Poetry: An International Anthology (pp. 25-44). Bristol: Intellect.

Thayer, P. (2009). Sleep.

Upton, B. (2015). The Aesthetic of Play. Cambridge, MA: The MIT Press.

Weizenbaum, J. (1976). Computer Power and Human Reason: From Judgment to Calculation. San Francisco, CA: W. H. Freeman and Company. 
Zunshine, L. (2006). Why We Read Fiction: Theory of Mind and the Novel. Columbus, OH: Ohio State University Press. 